\title{
15
}

\section{Diffusion processes and coalescent trees}

\author{
Robert C. Griffiths $\stackrel{a}{-}$ and Dario Spanób
}

\begin{abstract}
We dedicate this paper to Sir John Kingman on his 70th Birthday.

In modern mathematical population genetics the ancestral history of a population of genes back in time is described by John Kingman's coalescent tree. Classical and modern approaches model gene frequencies by diffusion processes. This paper, which is partly a review, discusses how coalescent processes are dual to diffusion processes in an analytic and probabilistic sense.

Bochner (1954) and Gasper (1972) were interested in characterizations of processes with Beta stationary distributions and Jacobi polynomial eigenfunctions. We discuss the connection with Wright-Fisher diffusions and the characterization of these processes. Subordinated Wright-Fisher diffusions are of this type. An Inverse Gaussian subordinator is interesting and important in subordinated Wright-Fisher diffusions and is related to the Jacobi Poisson Kernel in orthogonal polynomial theory. A related time-subordinated forest of non-mutant edges in the Kingman coalescent is novel.
\end{abstract}

AMS subject classification (MSC2010) 92D25, 60J70, 92D15

a Department of Statistics, University of Oxford, 1 South Parks Rd, Oxford OX1 3TG; griff@stats.ox.ac.uk

${ }^{b}$ Department of Statistics, University of Warwick, Coventry CV4 7AL;

D.Spano@warwick.ac.uk 


\section{Introduction}

The Wright-Fisher diffusion process $\{X(t), t \geq 0\}$ models the relative frequency of type $a$ genes in a population with two types of genes $a$ and $A$. Genes are subject to random drift and mutation over time. The generator of the process is

$$
\mathcal{L}=\frac{1}{2} x(1-x) \frac{\partial^{2}}{\partial x^{2}}+\frac{1}{2}(-\alpha x+\beta(1-x)) \frac{\partial}{\partial x},
$$

where the mutation rate $A \rightarrow a$ is $\frac{1}{2} \alpha$ and the rate $a \rightarrow A$ is $\frac{1}{2} \beta$. If $\alpha$ and $\beta$ are zero then zero and one are absorbing states where either $A$ or $a$ becomes fixed in the population. If $\alpha, \beta>0$ then $\{X(t), t \geq 0\}$ is a reversible process with a Beta stationary density

$$
f_{\alpha, \beta}(y)=B(\alpha, \beta)^{-1} y^{\alpha-1}(1-y)^{\beta-1}, 0<y<1 .
$$

The transition density has an eigenfunction expansion

$$
f(x, y ; t)=f_{\alpha, \beta}(y)\left\{1+\sum_{n=1}^{\infty} \rho_{n}^{\theta}(t) \widetilde{P}_{n}^{(\alpha, \beta)}(x) \widetilde{P}_{n}^{(\alpha, \beta)}(y)\right\},
$$

where $\theta=\alpha+\beta$,

$$
\rho_{n}^{\theta}(t)=\exp \left\{-\frac{1}{2} n(n+\theta-1) t\right\}
$$

and $\left\{\widetilde{P}_{n}^{(\alpha, \beta)}(y), n \in \mathbb{Z}_{+}\right\}$are orthonormal Jacobi polynomials on the Beta $(\alpha, \beta)$ distribution, scaled so that

$$
\mathbb{E}\left[\widetilde{P}_{m}^{(\alpha, \beta)}(Y) \widetilde{P}_{n}^{(\alpha, \beta)}(Y)\right]=\delta_{m n}, m, n \in \mathbb{Z}_{+}
$$

under the stationary distribution (1.2). The Wright-Fisher diffusion is also known as the Jacobi diffusion because of the eigenfunction expansion (1.3). The classical Jacobi polynomials, orthogonal on

$$
(1-x)^{\alpha}(1+x)^{\beta},-1<x<1,
$$

can be expressed as

$$
P_{n}^{(\alpha, \beta)}(x)=\frac{(\alpha+1)_{(n)}}{n !}{ }_{2} F_{1}(-n, n+\alpha+\beta+1 ; \alpha+1 ;(1-x) / 2),
$$

where ${ }_{2} F_{1}$ is a hypergeometric function. The relationship between the two sets of polynomials is that

$$
\widetilde{P}_{n}^{(\alpha, \beta)}(x)=c_{n} P_{n}^{(\beta-1, \alpha-1)}(2 x-1),
$$


where

$$
c_{n}=\sqrt{\frac{(2 n+\alpha+\beta-1)(\alpha+\beta)_{(n-1)} n !}{\alpha_{(n)} \beta_{(n)}}} .
$$

Define

$$
\overline{\mathcal{L}}=\frac{1}{2} \frac{\partial^{2}}{\partial x^{2}} x(1-x)-\frac{\partial}{\partial x} \frac{1}{2}(-\alpha x+\beta(1-x)),
$$

the forward generator of the process. The Jacobi polynomials are eigenfunctions satisfying, for $n \in \mathbb{Z}_{+}$,

$$
\begin{aligned}
\mathcal{L} \widetilde{P}_{n}^{(\alpha, \beta)}(x) & =-\frac{1}{2} n(n+\theta-1) \widetilde{P}_{n}^{(\alpha, \beta)}(x) \\
\overline{\mathcal{L}} f_{\alpha, \beta}(x) \widetilde{P}_{n}^{(\alpha, \beta)}(x) & =-\frac{1}{2} n(n+\theta-1) f_{\alpha, \beta}(x) \widetilde{P}_{n}^{(\alpha, \beta)}(x) .
\end{aligned}
$$

The well known fact that the Jacobi polynomials $\left\{\widetilde{P}_{n}^{(\alpha, \beta)}(x)\right\}$ satisfy (1.7) implies that they are eigenfunctions with corresponding eigenvalues $\left\{\rho_{n}^{\theta}(t)\right\}$.

In modern mathematical population genetics the ancestral history of a population back in time is described by John Kingman's elegant coalescent process [19]. The connection between the coalescent and FlemingViot diffusion processes is made explicit by Donnelly and Kurtz in |7], [8] by their look-down process. An approach by Ethier and Griffiths [10] uses duality to show that a 'non-mutant lines of descent' process which considers a forest of trees back in time to their first mutations is dual to the Fleming-Viot infinitely-many-alleles diffusion process. The twoallele process $\{X(t), t \geq 0\}$ is recovered from the Fleming-Viot process by a 2-colouring of alleles in the infinitely-many-alleles model. If there is no mutation then the dual process is the same as the Kingman coalescent process with an entrance boundary at infinity. The dual process approach leads to a transition density expansion in terms of the transition functions of the process which counts the number of non-mutant lineages back in time. It is interesting to make a connection between the eigenfunction expansion (1.3) and dual process expansion of the transition densities of $\{X(t), t \geq 0\}$. Bochner [6] and Gasper [13] find characterizations of processes which have Beta stationary distributions and Jacobi polynomial eigenfunctions. Subordinated Jacobi processes $\{X(Z(t)), t \geq 0\}$, where $\{Z(t), t \geq 0\}$ is a Lévy process, fit into this class, because subordination does not change the eigenvectors or the stationary distribution of the process. The subordinated processes are jump diffusions. A particular class of importance is when $\{Z(t), t \geq 0\}$ 
is an Inverse Gaussian process. Griffiths [18] obtains characterizations of processes with stationary distributions in the Meixner class, as well as for Jacobi processes. The current paper is partly a review paper describing connections between Jacobi diffusions, eigenfunction expansions of transition functions, coalescent trees, and Bochner characterizations. Novel results describe the subordinated non-mutant lines-of-descent process when the subordination is with an Inverse Gaussian process.

\section{A coalescent dual process}

A second form of the transition density (1.3) derived in Ethier and Griffiths [10] is

$$
f(x, y ; t)=\sum_{k=0}^{\infty} q_{k}^{\theta}(t) \sum_{l=0}^{k} \mathcal{B}(l ; k, x) f_{\alpha+l, \beta+k-l}(y)
$$

where

$$
\mathcal{B}(l ; k, x)=\left(\begin{array}{l}
l \\
k
\end{array}\right) x^{k}(1-x)^{l-k}, k=0,1, \ldots, l
$$

is the Binomial distribution and $\left\{q_{k}^{\theta}(t)\right\}$ are the transition functions of a death process with an entrance boundary of infinity, and death rates $k(k+\theta-1) / 2, k \geq 1$. The death process represents the number of nonmutant ancestral lineages back in time in the coalescent process with mutation. The number of lineages decreases from $k$ to $k-1$ from coalescence at rate $\left(\begin{array}{l}k \\ 2\end{array}\right)$ or mutation at rate $k \theta / 2$. If there is no mutation, $\left\{q_{k}^{0}(t), t \geq 0\right\}$ are transition functions of the number of edges in a Kingman coalescent tree. There is an explicit expression for the transition functions beginning with the entrance boundary of infinity [16, 21, 17] of

$$
q_{k}^{\theta}(t)=\sum_{j=k}^{\infty} \rho_{j}^{\theta}(t)(-1)^{j-k} \frac{(2 j+\theta-1)(k+\theta)_{(j-1)}}{k !(j-k) !},
$$

recalling that $\rho_{n}^{\theta}(t)$ is defined by (1.4). A complex-variable representation of (2.2) is found in [17]. Let $\left\{X_{t}, t \geq 0\right\}$ be standard Brownian motion so $X_{t}$ is $N(0, t)$. Denote $Z_{t}=e^{i X_{t}}$ and $\omega_{t}=e^{-\frac{1}{2} \theta t}$, then

$$
q_{k}^{\theta}(t)=e^{\frac{1}{8} t} \frac{\Gamma(2 k+\theta)}{\Gamma(k+\theta) k !} \mathbb{E}\left[\frac{\left(\omega_{t} Z_{t}\right)^{k}\left(1-\omega_{t} Z_{t}\right)}{\sqrt{Z_{t}}\left(1+\omega_{t} Z_{t}\right)^{2 k+\theta}}\right],
$$


for $k=0,1, \ldots$ The transition functions for the process beginning at $n$, rather than infinity, are

$$
q_{n k}^{\theta}(t)=\sum_{j=k}^{n} \rho_{j}^{\theta}(t)(-1)^{j-k} \frac{(2 j+\theta-1)(k+\theta)_{(j-1)} n_{[j]}}{k !(j-k) !(n+\theta)_{(j)}},
$$

for $k=0,1, \ldots, n$. An analogous complex-variable representation to $(2.3)$ is

$$
\begin{gathered}
q_{n k}^{\theta}(t)=\frac{\Gamma(n+\theta) \Gamma(2 k+\theta)}{\Gamma(k+\theta) \Gamma(n+k+\theta)}\left(\begin{array}{l}
n \\
k
\end{array}\right) e^{\frac{1}{8}(\theta-1)^{2} t} \mathbb{E}\left[Z_{t}^{k+(\theta-1) / 2}\left(1-Z_{t}\right)\right. \\
\left.\times{ }_{2} F_{1}\left(-n+k+1, \theta+2 k ; n+k+\theta ; Z_{t}\right)\right]
\end{gathered}
$$

for $k=0,1, \ldots, n$. The expansion (2.1) is derived from a two-dimensional dual death process $\left\{L^{\theta}(t) \in \mathbb{Z}_{+}^{2}, t \geq 0\right\}$ which looks back in time in the diffusion process $\{X(t), t \geq 0\}$. A derivation in this paper is from [9], which follows more general analytic derivations in [10] for a FlemingViot model and 3] for a diffusion model with selection. Etheridge and Griffiths [9] give a very clear probabilistic derivation in a Moran model with selection that provides an understanding of earlier derivations. A sketch of a derivation of (2.1) from [9] is the following. Let $x_{1}=x$, $x_{2}=1-x$ and define for $k \in \mathbb{Z}_{+}^{2}$

$$
g_{k}(x)=\frac{\theta_{(|k|)}}{\alpha_{\left(k_{1}\right)} \beta_{\left(k_{2}\right)}} x_{1}^{k_{1}} x_{2}^{k_{2}}
$$

then

$$
\mathcal{L} g_{k}(x)=\frac{1}{2}(|k|+\theta-1)\left[k_{1} g_{k-e_{1}}(x)+k_{2} g_{k-e_{2}}(x)-|k| g_{k}(x)\right] .
$$

Here and elsewhere we use the notation $|y|=\sum_{j=1}^{d} y_{j}$ for a $d$-dimensional vector $y$. In this particular case $|k|=k_{1}+k_{2}$. To obtain a dual process the generator is regarded as acting on $k=\left(k_{1}, k_{2}\right)$, rather than $x$. The dual process is a two-dimensional death process $\left\{L^{\theta}(t), t \geq 0\right\}$, the rates of which are read off from the coefficients of the functions $g$ on the right-hand side of (2.6);

$$
k \rightarrow k-e_{i} \quad \text { at rate } \quad \frac{1}{2} \frac{k_{i}}{|k|} \cdot|k|(|k|+\theta-1) .
$$

The total size, $\left|L^{\theta}(t)\right|$, is a 1-dimensional death process in which

$$
|k| \rightarrow|k|-1 \quad \text { at rate } \frac{1}{2}|k|(|k|+\theta-1)
$$


with transition functions denoted by $\left\{q_{m l}^{\theta}(t), t \geq 0\right\}$. There is hypergeometric sampling of types which do not die, so

$$
P(L(t)=l \mid L(0)=m)=q_{m l}^{\theta}(t)=q_{|m||l|}^{\theta}(t) \frac{\left(\begin{array}{c}
m_{1} \\
l_{1}
\end{array}\right)\left(\begin{array}{c}
m_{2} \\
l_{2}
\end{array}\right)}{\left(\begin{array}{c}
\mid \\
m
\end{array}\right)|| l \mid},
$$

where $q_{|m||l|}^{\theta}(t)$ is defined in (2.4). The dual equation obtained by regarding $\mathcal{L}$ as acting on $x$ or $k$ in (2.6) is

$$
\mathbb{E}_{X(0)}\left[g_{L(0)}(X(t))\right]=\mathbb{E}_{L(0)}\left[g_{L(t)}(X(0))\right]
$$

where expectation on the left is with respect to the distribution of $X(t)$, and on the right with respect to the distribution of $L(t)$. Partitioning the expectation on the right of (2.9) by values taken by $L(t)$,

$$
\begin{aligned}
& \mathbb{E}_{x}\left[\left(\begin{array}{c}
|m| \\
m_{1}
\end{array}\right) X_{1}(t)^{m_{1}} X_{2}(t)^{m_{2}}\right] \\
& =\left(\begin{array}{c}
|m| \\
m_{1}
\end{array}\right) \frac{\alpha_{\left(m_{1}\right)} \beta_{\left(m_{2}\right)}}{\theta_{\left(m_{1}+m_{2}\right)}} \sum_{l \leq m} x_{1}^{l_{1}} x_{2}^{l_{2}} \frac{\theta_{(|l|)}}{\alpha_{\left(l_{1}\right)} \beta_{\left(l_{2}\right)}} q_{|m||l|}^{\theta}(t)\left(\begin{array}{c}
|l| \\
l_{1}
\end{array}\right) \frac{m_{1\left[l_{1}\right]} m_{2\left[l_{2}\right]}}{|m|_{[|l|]}} .
\end{aligned}
$$

The transition distribution of $X(t)$ now has an expansion derived from an inversion formula applied to (2.10). Letting $m_{1}, m_{2} \rightarrow \infty$ with $m_{1} /|m| \rightarrow y_{1}, m_{2} /|m| \rightarrow y_{2}$ gives

$$
f(x, y ; t)=\sum_{l \in \mathbb{Z}_{+}^{2}} q_{|l|}^{\theta}(t)\left(\begin{array}{c}
|l| \\
l_{1}
\end{array}\right) x_{1}^{l_{1}} x_{2}^{l_{2}} B\left(\alpha+l_{1}, \beta+l_{2}\right)^{-1} y_{1}^{l_{1}+\alpha-1} y_{2}^{l_{2}+\beta-1}
$$

which is identical to (2.1).

The two-allele Wright-Fisher diffusion is a special case of a much more general Fleming-Viot measure-valued diffusion process which has $\mathcal{P}(S)$, the probability measures on $S$, a compact metric space, as a state space. The mutation operator in the process is

$$
(A f)(x)=\frac{\theta}{2} \int_{S}(f(\xi)-f(x)) \nu_{0}(d \xi),
$$

where $\nu_{0} \in \mathcal{P}(S)$ and $f: S \rightarrow \mathbb{R}$. The stationary measure is a PoissonDirichlet (Ferguson-Dirichlet) random measure

$$
\mu=\sum_{i=1}^{\infty} x_{i} \delta_{\xi_{i}}
$$

where $\left\{x_{i}\right\}$ is a Poisson-Dirichlet point process, $\mathcal{P} \mathcal{D}(\theta)$, independent of $\left\{\xi_{j}\right\}$ which are i.i.d. $\nu_{0} \in \mathcal{P}(S)$. A description of the $\mathcal{P} \mathcal{D}(\theta)$ distribution is contained in Kingman [20]. 
Denote the stationary distribution of the random measure as

$$
\Pi_{\theta, \nu_{0}}(\cdot)=\mathbb{P}(\mu \in \cdot) .
$$

Ethier and Griffiths [10] derive a transition function expansion for $P(t, \mu$, $d \nu)$ with given initial $\mu \in \mathcal{P}(S)$ of

$$
\begin{aligned}
\mathbb{P}(t, \mu, .)=q_{0}^{\theta}(t) \prod_{\theta, \nu_{0}}(\cdot) & \\
+ & +\sum_{n=1}^{\infty} q_{n}^{\theta}(t) \int_{S^{n}} \mu^{n}\left(d x_{1} \times \cdots \times d x_{n}\right) \\
& \times \prod_{n+\theta,(n+\theta)^{-1}\left\{n \eta_{n}\left(x_{1}, \ldots, x_{n}\right)+\theta \nu_{0}\right\}}(\cdot),
\end{aligned}
$$

where $\eta_{n}\left(x_{1}, \ldots, x_{n}\right)$ is the empirical measure of points $x_{1}, \ldots, x_{n} \in S$ :

$$
\eta_{n}\left(x_{1}, \ldots, x_{n}\right)=n^{-1}\left(\delta_{x_{1}}+\cdots+\delta_{x_{n}}\right) .
$$

There is the famous Kingman coalescent process tree [19] behind the pretty representation (2.11). The coalescent tree has an entrance boundary at infinity and a coalescence rate of $\left(\begin{array}{l}k \\ 2\end{array}\right)$ while there are $k$ edges in the tree. Mutations occur according to a Poisson process of rate $\theta / 2$ along the edges of the coalescent tree. $\left\{q_{n}^{\theta}(t)\right\}$ is the distribution of the number of non-mutant edges in the tree at time $t$ back. The number of non-mutant edges is the same as the number of edges in a forest where coalescence occurs to non-mutant edges and trees are rooted back in time when mutations occur on an edge. If the time origin is at time $t$ back and there are $n$ non-mutant edges at the origin then the leaves of the infinite-leaf tree represent the population at $t$ forward in time divided into relative frequencies of families of types which are either the $n$ non-mutant types chosen at random from time zero, or mutant types chosen from $\nu_{0}$ in $(0, t)$. The frequencies of non-mutant families, scaled to have a total frequency unity, have a Dirichlet distribution with unit index parameters, and the new mutation families, scaled to have total frequency unity, are distributed according to a Poisson-Dirichlet random measure with rate $\theta$ and type measure $\nu_{0}$. The total frequency of new mutations has a Beta $(\theta, n-1)$ distribution. An extended description of the tree process is in Griffiths [17].

A $d$-dimensional reversible diffusion process model for gene frequencies which arises as a limit from the Wright-Fisher model has a backward generator

$$
\mathcal{L}=\frac{1}{2} \sum_{i=1}^{d} \sum_{j=1}^{d} x_{i}\left(\delta_{i j}-x_{j}\right) \frac{\partial^{2}}{\partial x_{i} \partial x_{j}}+\frac{1}{2} \sum_{i=1}^{d}\left(\epsilon_{i}-\theta x_{i}\right) \frac{\partial}{\partial x_{i}}
$$


where $\theta=|\epsilon|$. In this model mutation is parent-independent from type $i \rightarrow j$ at rate $\frac{1}{2} \epsilon_{j}, i, j=1, \ldots, d$. Assuming that $\epsilon>0$, the stationary density is the Dirichlet density

$$
\frac{\Gamma(\theta)}{\Gamma\left(\epsilon_{1}\right) \cdots \Gamma\left(\epsilon_{d}\right)} x_{1}^{\epsilon_{1}-1} \cdots x_{d}^{\epsilon_{d}-1}
$$

for $x_{1}, \ldots, x_{d}>0$ and $\sum_{1}^{d} x_{i}=1$. Griffiths [15] shows that the transition density in the model has eigenvalues

$$
\rho_{|n|}(t)=e^{-\frac{1}{2}|n|(|n|+\theta-1) t}
$$

repeated

$$
\left(\begin{array}{c}
|n|+d-2 \\
|n|
\end{array}\right)
$$

times corresponding to eigenvectors $\left\{Q_{n}^{\circ}(x), n \in \mathbb{Z}_{+}^{d-1}\right\}$ which are multitype orthonormal polynomials of total degree $|n|$ in $x$. As eigenfunctions the polynomials satisfy

$$
\mathcal{L} Q_{n}^{\circ}(x)=-\frac{1}{2}|n|(|n|+\theta-1) Q_{n}^{\circ}(x) .
$$

The eigenvalues $\left\{\rho_{k}(t), k \in \mathbb{Z}_{+}\right\}$do not depend on the dimension $d$. The transition density with $X(0)=x, X(t)=y$ has the form

$$
f(x, y, t)=\mathcal{D}(y, \epsilon)\left\{1+\sum_{|n|=1}^{\infty} \rho_{|n|}(t) Q_{|n|}(x, y)\right\} .
$$

The kernel polynomials on the Dirichlet $\left\{Q_{|n|}(x, y)\right\}$ appearing in (2.15) are defined as

$$
Q_{|n|}(x, y)=\sum_{\{n:|n| \text { fixed }\}} Q_{n}^{\circ}(x) Q_{n}^{\circ}(y)
$$

for any complete orthonormal polynomial set $\left\{Q_{n}^{\circ}(x)\right\}$ on the Dirichlet distribution (2.13). If $d=2$,

$$
Q_{|n|}(x, y)=\widetilde{P}_{|n|}^{\left(\epsilon_{1}, \epsilon_{2}\right)}(x) \widetilde{P}_{|n|}^{\left(\epsilon_{1}, \epsilon_{2}\right)}(y)
$$

where $\left\{\widetilde{P}_{|n|}^{\left(\epsilon_{1}, \epsilon_{2}\right)}(x)\right\}$ are orthonormal Jacobi polynomials on the Beta distribution on $[0,1]$. In general $n$ is just a convenient index system for the polynomials since the number of polynomials of total degree $|n|$ is always the same as the number of solutions of $n_{1}+\cdots+n_{d-1}=|n|$,

$$
\left(\begin{array}{c}
|n|+d-2 \\
|n|
\end{array}\right)
$$


$Q_{|n|}(x, y)$ is invariant under the choice of which orthonormal polynomial set is used. The individual polynomials $Q_{n}^{\circ}(x)$ are uniquely determined by their leading coefficients of degree $|n|$ and $Q_{|n|}(x, y)$. A specific form is

$$
Q_{|n|}(x, y)=(\theta+2|n|-1) \sum_{m=0}^{|n|}(-1)^{|n|-m} \frac{(\theta+m)_{(|n|-1)}}{m !(|n|-m) !} \xi_{m}
$$

where

$$
\xi_{m}=\sum_{|l|=m}\left(\begin{array}{c}
m \\
l
\end{array}\right) \frac{\theta_{(m)}}{\prod_{1}^{d} \epsilon_{i\left(l_{i}\right)}} \prod_{1}^{d}\left(x_{i} y_{i}\right)^{l_{i}} .
$$

An inverse relationship is

$$
\xi_{m}=1+\sum_{|n|=1}^{m} \frac{m_{[|n|]}}{(\theta+m)_{(|n|)}} Q_{|n|}(x, y) .
$$

The transition distribution (2.15) is still valid if any or all elements of $\epsilon$ are zero. The constant term in the expansion then vanishes as the diffusion process is transient and there is not a stationary distribution. For example, if $\epsilon=0$,

$$
f(x, y, t)=\prod_{j=1}^{d} y_{j}^{-1}\left\{\sum_{|n| \geq d}^{\infty} \rho_{|n|}(t) Q_{|n|}^{0}(x, y)\right\}
$$

where

$$
Q_{|n|}^{0}(x, y)=(2|n|-1) \sum_{m=1}^{n}(-1)^{|n|-m} \frac{(m)_{(|n|-1)}}{m !(|n|-m) !} \xi_{m}^{0},
$$

with

$$
\xi_{m}^{0}=\sum_{\{l: l>0,|l|=m\}}\left(\begin{array}{c}
m \\
l
\end{array}\right) \frac{(m-1) !}{\prod_{1}^{d}\left(l_{i}-1\right) !} \prod_{1}^{d}\left(x_{i} y_{i}\right)^{l_{i}} .
$$

The derivation of (2.15) is a very classical approach. The same process can be thought of as arising from an infinite-leaf coalescent tree similar to the description in the Fleming-Viot infinitely-many-alleles process. The coalescent rate while there are $k$ edges in the tree is $\left(\begin{array}{l}k \\ 2\end{array}\right)$ and mutations occur along edges at rate $\theta / 2$. In this model there are $d$ types, $1,2, \ldots$, $d$ and the probability of mutation $i \rightarrow j$, given a mutation, is $\epsilon_{j} / \theta$. This is equivalent to a $d$-colouring of alleles in the Fleming-Viot infinitelymany-alleles model. Think backwards from time $t$ back to time 0 . Let $y=\left(y_{1}, \ldots, y_{d}\right)$ be the relative frequencies of types in the infinite number 
of leaves at the current time $t$ forward and $x=\left(x_{1}, \ldots, x_{d}\right)$ be the frequencies in the population at time 0 . Let $l$ be the number of nonmutant edges at time 0 which have families at time $t$ in the leaves of the tree. Given these $l$ edges let $U=\left(U_{1}, \ldots, U_{l}\right)$ be their relative family sizes in the leaves, and $V=\left(V_{1}, \ldots, V_{d}\right)$ be the frequencies of families derived from new mutations on the tree edges in $(0, t)$. The distribution of $U \oplus V=\left(U_{1}, \ldots, U_{l}, V_{1}, \ldots, V_{d}\right)$ is $\mathcal{D}(u \oplus v,(1, \ldots, 1) \oplus \epsilon)$. The type of the $l$ lines, and therefore their families, is chosen at random from the frequencies $x$. The distribution of the number of non-mutant lines at time 0 from the population at $t$ is $q_{l}^{\theta}(t)$. The transition density in the diffusion (2.15) is identical to the mixture distribution arising from the coalescent

$$
f(x, y, t)=\sum_{|l|=0}^{\infty} q_{|l|}^{\theta}(t) \sum_{\{l:|l| \text { fixed }\}} \mathcal{M}(l, x) \mathcal{D}(y, \epsilon+l),
$$

by considering types of non-mutant lines, and adding Dirichlet variables and parameters according to $l_{i}$ non-mutant families being of type $i$. $\mathcal{M}(l, x)$ is the multinomial distribution describing the choice of the initial line types from the population at time 0 . The expansion when $d=2$ corresponds to (1.3). The argument is valid if any elements of $\epsilon$ are zero, considering a generalized Dirichlet distribution $\mathcal{D}(x, \epsilon)$ where if $\epsilon_{i}=0$, then $X_{i}=0$ with probability 1 .

The algebraic identity of (2.23) and (2.15) is easy to see by expressing $Q_{|n|}(x, y)$ in terms of $\left\{\xi_{m}\right\}$, then collecting coefficients of $\xi_{|l|}$ in (2.15) to obtain (2.23). Setting $\rho_{0}(t)=1$ and $Q_{0}(x, y)=1$, the transition density is

$$
\begin{aligned}
f(x, y, t) & =\mathcal{D}(y, \epsilon) \sum_{|n|=0}^{\infty} \rho_{|n|}(t) Q_{|n|}(x, y) \\
& =\sum_{l \in \mathbb{Z}_{+}^{d}}^{\infty}\left[\sum_{|n|=|l|}^{\infty} \rho_{|n|}(t)(\theta+2|n|-1)(-1)^{|n|-|l|} \frac{(\theta+|l|)_{(|n|-1)}}{|l| !(|n|-|l|) !}\right] \\
& \times \mathcal{D}(y, \epsilon) \xi_{l}(x, y) \\
= & \sum_{|l|=0}^{\infty} q_{|l|}^{\theta}(t) \sum_{\{l:|l| \text { fixed }\}} \mathcal{M}(l, x) \mathcal{D}(y, \epsilon+l) .
\end{aligned}
$$

The non-mutant line-of-descent process with transition probabilities $\left\{q_{n}^{\theta}(t)\right\}$ appears in all the Wright-Fisher diffusion processes mentioned in this section as a fundamental dual process. The process does not depend on the dimension of the diffusion, partly because the $d$-dimensional 
process can be recovered from the measure-valued process as a special case by colouring new mutations into $d$ classes with probabilities $\left(\epsilon_{1} / \theta, \epsilon_{2} / \theta, \ldots, \epsilon_{d} / \theta\right)$ with $\theta=\sum_{j=1}^{d} \epsilon_{j}$. It is also interesting to see the derivation of the $d$-dimensional transition density expansion as a mixture in terms of $\left\{q_{n}^{\theta}(t)\right\}$ via the orthogonal-function expansion of the transition density in (2.24).

\section{Processes with beta stationary distributions and Jacobi polynomial eigenfunctions}

In this section we consider 1-dimensional processes which have Beta stationary distributions and Jacobi polynomial eigenfunctions, and their connection with Wright-Fisher diffusion processes. We begin by considering Bochner [6] and Gasper's [13] characterization of bivariate Beta distributions.

A class of bivariate distributions with Beta marginals and Jacobi polynomial eigenfunctions has the form

$$
f(x, y)=f_{\alpha \beta}(x) f_{\alpha \beta}(y)\left\{1+\sum_{n=1}^{\infty} \rho_{n} \widetilde{P}_{n}^{(\alpha, \beta)}(x) \widetilde{P}_{n}^{(\alpha, \beta)}(y)\right\},
$$

where $\left\{\rho_{n}, n \in \mathbb{Z}_{+}\right\}$is called a correlation sequence. The transition density (1.3) in the Jacobi diffusion has the form of the conditional density of $Y$ given $X=x$ in (3.1) with $\rho_{n} \equiv \rho_{n}^{\theta}(t)$. Bochner [6] and Gasper [13] worked on characterizations of sequences $\left\{\rho_{n}\right\}$ such that the expansion (3.1) is positive, and thus a probability distribution. It is convenient to normalize the Jacobi polynomials by taking

$$
R_{n}^{(\alpha, \beta)}(x)=\frac{\widetilde{P}_{n}^{(\alpha, \beta)}(x)}{\widetilde{P}_{n}^{(\alpha, \beta)}(1)}
$$

so that $R_{n}^{(\alpha, \beta)}(1)=1$; denote

$$
h_{n}^{-1}=\mathbb{E}\left[R_{n}^{(\alpha, \beta)}(X)^{2}\right]=\frac{(2 n+\alpha+\beta-1)(\alpha+\beta)_{(n-1)} \beta_{(n)}}{\alpha_{(n)} n !},
$$

and write

$$
f(x, y)=f_{\alpha \beta}(x) f_{\alpha \beta}(y)\left\{1+\sum_{n=1}^{\infty} \rho_{n} h_{n} R_{n}^{(\alpha, \beta)}(x) R_{n}^{(\alpha, \beta)}(y)\right\} .
$$

Bochner [6] defined a bounded sequence $\left\{c_{n}\right\}$ to be positive definite with 
respect to the Jacobi polynomials if

$$
\sum_{n \geq 0} a_{n} h_{n} R_{n}^{(\alpha, \beta)}(x) \geq 0, \quad \sum_{n \geq 0}\left|a_{n}\right| h_{n}<\infty
$$

implies that

$$
\sum_{n \geq 0} a_{n} c_{n} h_{n} R_{n}^{(\alpha, \beta)}(x) \geq 0 .
$$

Then $\left\{\rho_{n}\right\}$ is a correlation sequence if and only if it is a positive definite sequence. The only if proof follows from

$$
\sum_{n \geq 0} a_{n} \rho_{n} R_{n}^{(\alpha, \beta)}(x)=\mathbb{E}\left[\sum_{n \geq 0} a_{n} h_{n} R_{n}^{(\alpha, \beta)}(Y) \mid X=x\right] \geq 0,
$$

where $(X, Y)$ has the distribution (3.2). The if proof follows at least heuristically by noting that

$$
\sum_{n \geq 0} h_{n} R_{n}^{(\alpha, \beta)}(x) R_{n}^{(\alpha, \beta)}(y)=\frac{\delta(x-y)}{f_{\alpha, \beta}(x)} \geq 0,
$$

where $\delta(\cdot)$ has a unit point mass at zero, so if $\left\{\rho_{n}\right\}$ is a positive definite sequence then

$$
\sum_{n \geq 0} \rho_{n} h_{n} R_{n}^{(\alpha, \beta)}(x) R_{n}^{(\alpha, \beta)}(y) \geq 0
$$

and (3.2) is non-negative. A careful proof is given in [14].

Under the conditions that

$$
\alpha<\beta \text { and either } 1 / 2 \leq \alpha \text { or } \alpha+\beta \geq 2,
$$

it is shown in 13] that a sequence $\rho_{n}$ is positive definite if and only if

$$
\rho_{n}=\mathbb{E}\left[R_{n}^{(\alpha, \beta)}(Z)\right]
$$

for some random variable $Z$ in $[0,1]$. If the conditions (3.3) do not hold then there exist $x, y, z$ such that $K(x, y, z)<0$. The sufficiency rests on showing that under the conditions (3.3) for $x, y, z \in[0,1]$,

$$
K(x, y, z)=\sum_{n=0}^{\infty} h_{n} R_{n}^{(\alpha, \beta)}(x) R_{n}^{(\alpha, \beta)}(y) R_{n}^{(\alpha, \beta)}(z) \geq 0 .
$$

The sufficiency of (3.4) is then clear by mixing over a distribution for $Z$ in (3.5) to get positivity. The necessity follows by setting $x=1$ in

$$
\rho_{n} R_{n}^{(\alpha, \beta)}(x)=\mathbb{E}\left[R_{n}^{(\alpha, \beta)}(Y) \mid X=x\right],
$$

and recalling that $R_{n}^{(\alpha, \beta)}(1)=1$, so that $Z$ is distributed as $Y$ conditional 
on $X=1$. This implies that extreme correlation sequences in exchangeable bivariate Beta distributions with Jacobi polynomial eigenfunctions are the scaled Jacobi polynomials $\left\{R_{n}^{(\alpha, \beta)}(z), z \in[0,1]\right\}$. Bochner [6] was the original author to consider such problems for the ultraspherical polynomials, essentially orthogonal polynomials on Beta distributions with equal parameters.

A characterization of reversible Markov processes with stationary Beta distribution and Jacobi polynomial eigenfunctions, from [13], under (3.3), is that they have transition functions of the form

$$
f(x, y ; t)=f_{\alpha \beta}(y)\left\{1+\sum_{n=1}^{\infty} c_{n}(t) h_{n} R_{n}^{(\alpha, \beta)}(x) R_{n}^{(\alpha, \beta)}(y)\right\},
$$

with $c_{n}(t)=\exp \left\{-d_{n} t\right\}$, where

$$
d_{n}=\sigma n(n+\alpha+\beta-1)+\int_{0}^{1-} \frac{1-R_{n}^{(\alpha, \beta)}(z)}{1-z} \nu(d z),
$$

$\sigma \geq 0$, and $\nu$ is a finite measure on $[0,1)$. If $\nu(\cdot) \equiv 0$, a null measure, then $f(x, y ; t)$ is the transition function of a Jacobi diffusion.

Eigenvalues of a general reversible time-homogeneous Markov process with countable spectrum must satisfy Bochner's consistency conditions:

(i) $\left\{c_{n}(t)\right\}$ is a correlation sequence for each $t \geq 0$,

(ii) $c_{n}(t)$ is continuous in $t \geq 0$,

(iii) $c_{n}(0)=c_{0}(t)=1$, and

(iv) $c_{n}(t+s)=c_{n}(t) c_{n}(s)$ for $t, s \geq 0$.

If there is a spectrum $\left\{c_{n}(t)\right\}$ with corresponding eigenfunctions $\left\{\xi_{n}\right\}$ then

$$
\begin{aligned}
c_{n}(t+s) \xi_{n}(X(0)) & =\mathbb{E}\left[\xi_{n}(X(t+s)) \mid X(0)\right] \\
& =\mathbb{E}\left[\mathbb{E}\left[\xi_{n}(X(t+s)) \mid X(s)\right] \mid X(0)\right] \\
& =c_{n}(t) \mathbb{E}\left[\xi_{n}(X(s)) \mid X(0)\right] \\
& =c_{n}(t) c_{n}(s) \xi_{n}(X(0)),
\end{aligned}
$$

showing (iv). If a stationary distribution exists and $X(0)$ has this distribution then the eigenfunctions can be scaled to be orthonormal on this distribution and the eigenfunction property is then

$$
\mathbb{E}\left[\xi_{m}(X(t)) \xi_{n}(X(0))\right]=c_{n}(t) \delta_{m n}
$$


$\{X(t), t \geq 0\}$ is a Markov process such that the transition distribution of $Y=X(t)$ given $X(0)=x$ is

$$
f(x, y ; t)=f(y)\left\{1+\sum_{n=1}^{\infty} c_{n}(t) \xi_{n}(x) \xi_{n}(y)\right\},
$$

where $f(y)$ is the stationary distribution. In our context $\left\{\xi_{n}\right\}$ are the orthonormal Jacobi polynomials. A Jacobi process $\{X(t), t \geq 0\}$ with transition distributions (3.6) can be constructed in the following way, which is analogous to constructing a general Lévy process from a compound Poisson process. Let $\left\{X_{k}, k \in \mathbb{Z}_{+}\right\}$be a Markov chain with stationary distribution $f_{\alpha \beta}(y)$ and transition distribution of $Y$ given $X=x$ corresponding to (3.1), with (3.3) holding, and $\{N(t), t \geq 0\}$ be an independent Poisson process of rate $\lambda$. Then $\left(X_{0}, X_{k}\right)$ has a correlation sequence $\left\{\rho_{n}^{k}\right\}$ and the transition functions of $X(t)=X_{N(t)}$ have the form (3.8), with

$$
d_{n}=\lambda \int_{0}^{1}\left(1-R_{n}^{(\alpha, \beta)}(z)\right) \mu(d z)
$$

where $\mu$ is a probability measure on $[0,1]$. The general form (3.7) is obtained by choosing a pair $\left(\lambda, \mu_{\lambda}\right)$ such that

$$
d_{n}=\lim _{\lambda \rightarrow \infty} \lambda \int_{0}^{1}\left(1-R_{n}^{(\alpha, \beta)}(z)\right) \mu_{\lambda}(d z)=\int_{0}^{1} \frac{1-R_{n}^{(\alpha, \beta)}(z)}{1-z} \nu(d z) .
$$

Equation (3.10) agrees with (3.7) when any atom $\nu(\{1\})$ is taken out of the integral, because

$$
\lim _{z \rightarrow 1} \frac{1-R_{n}^{(\alpha, \beta)}(z)}{1-z}=\operatorname{cn}(n+\theta-1),
$$

where $c \geq 0$ is a constant.

\section{Subordinated Jacobi diffusion processes}

Let $\{X(t), t \geq 0\}$ be a process with transition functions (3.6), and $\{Z(t), t \geq 0\}$ be a non-negative Lévy process with Laplace transform

$$
\mathbb{E}\left[e^{-\lambda Z(t)}\right]=\exp \left\{-t \int_{0}^{\infty} \frac{1-e^{-\lambda y}}{y} H(d y)\right\},
$$

where $\lambda \geq 0$ and $H$ is a finite measure. The subordinated process $\{\widetilde{X}(t)=X(Z(t)), t \geq 0\}$ is a Markov process which belongs to the 
same class of processes with correlation sequences

$$
\widetilde{c}_{n}(t)=\mathbb{E}\left[c_{n}(Z(t))\right]=\exp \left\{-t \int_{0}^{\infty} \frac{1-e^{-d_{n} y}}{y} H(d y)\right\},
$$

where $H$ is a finite measure. $\widetilde{c}_{n}(t)$ necessarily has a representation as $e^{-\widetilde{d}_{n} t}$, where $\widetilde{d}_{n}$ has the form (3.10) for some measure $\widetilde{\nu}$. We describe the easiest case from which the general case can be obtained as a limit. Suppose

$$
\lambda=\int_{0}^{\infty} \frac{H(d y)}{y}<\infty,
$$

and write

$$
G(d y)=\frac{H(d y)}{\lambda y}
$$

so that $G$ is a probability measure. Let

$$
K(d z)=f_{\alpha \beta}(z) d z\left\{1+\sum_{n=1}^{\infty} h_{n} R_{n}^{(\alpha, \beta)}(z) \int_{0}^{\infty} e^{-d_{n} y} G(d y)\right\}
$$

Then $K$ is a probability measure and

$$
\lambda \int_{0}^{1}\left(1-R_{n}^{(\alpha, \beta)}(z)\right) K(d z)=\lambda \int_{0}^{\infty}\left(1-e^{-d_{n} y}\right) G(d y) .
$$

The representation (3.10) is now obtained by setting

$$
\widetilde{\nu}(d z)=\lambda(1-z) K(d z) .
$$

We now consider subordinated Jacobi diffusion processes. The subordinated process is no longer a diffusion process because $\{Z(t), t \geq 0\}$ is a jump process and therefore $\{\widetilde{X}(t), t \geq 0\}$ has discontinuous sample paths. It is possible to construct processes such that (4.2) holds with $d_{n}=n$ by showing that $e^{-t n}$ is a correlation sequence and thus so is $\mathbb{E}\left[e^{-Z(t) n}\right]$. The construction follows an idea in [6]. The Jacobi-Poisson kernel in orthogonal polynomial theory is

$$
1+\sum_{n=1}^{\infty} r^{n} h_{n} R_{n}^{(\alpha, \beta)}(x) R_{n}^{(\alpha, \beta)}(y)
$$

which is non-negative for all $\alpha, \beta>0, x, y \in[0,1]$, and $0 \leq r \leq 1$, for which see [1], p112. The series (4.3) is a classical one evaluated early in research on Jacobi polynomials (see [2]). In terms of the original Jacobi 
polynomials, (1.5)

$$
\begin{aligned}
& \sum_{n=0}^{\infty} r^{n} \phi_{n} P_{n}^{(\alpha, \beta)}(x) P_{n}^{(\alpha, \beta)}(y) \\
& =\frac{\Gamma(\alpha+\beta+2)(1-r)}{2^{\alpha+\beta+1} \Gamma(\alpha+1) \Gamma(\beta+1)(1+r)^{\alpha+\beta+2}} \\
& \times \sum_{m, n=0}^{\infty} \frac{((\alpha+\beta+2) / 2)_{(m+n)}((\alpha+\beta+3) / 2)_{(m+n)}}{(\alpha+1)_{(m)}(\beta+1)_{(m)} m ! n !}\left(\frac{a^{2}}{k^{2}}\right)^{m}\left(\frac{b^{2}}{k^{2}}\right)^{n},
\end{aligned}
$$

where

$$
\phi_{n}^{-1}=\frac{2^{\alpha+\beta+1}}{2 n+\alpha+\beta+1} \frac{\Gamma(n+\alpha+1) \Gamma(n+\beta+1)}{\Gamma(n+1) \Gamma(n+\alpha+\beta+1)},
$$

$x=\cos 2 \varphi, y=\cos 2 \theta, a=\sin \varphi \sin \theta, b=\cos \varphi \cos \theta, k=\left(r^{1 / 2}+\right.$ $\left.r^{-1 / 2}\right) / 2$. The series (4.4) is positive for $-1 \leq x, y \leq 1,0 \leq r<1$ and $\alpha, \beta>-1$.

A Markov process analogy to the Jacobi-Poisson kernel is when the eigenvalues $c_{n}(t)=\exp \{-n t\}$. Following [6] let $\widetilde{X}(t)=X(Z(t))$, where $\{Z(t), t \geq 0\}$ is a Lévy process with Laplace transform

$$
\begin{aligned}
\mathbb{E} & {\left[e^{-\lambda Z(t)}\right] } \\
& =\exp \left\{-t\left[\sqrt{2 \lambda+(\theta-1)^{2} / 4}-\sqrt{(\theta-1)^{2} / 4}\right]\right\} \\
& =\exp \left\{-\frac{t}{\sqrt{2 \pi}} \int_{0}^{\infty} \frac{e^{-x(\theta-1)^{2} / 8}}{x^{3 / 2}}\left(1-e^{-x \lambda}\right) d x\right\} .
\end{aligned}
$$

$\{Z(t), t \geq 0\}$ is a tilted positive stable process with index $\frac{1}{2}$ such that $Z(t)$ has an Inverse Gaussian density

$$
I G\left(\frac{2 t}{|\theta-1|}, t^{2}\right), \theta \neq 1
$$

that is,

$$
\frac{t}{\sqrt{2 \pi z^{3}}} \exp \left\{-\frac{1}{2 z}\left(\frac{|\theta-1|}{2} z-t\right)^{2}\right\}, z>0 .
$$

The usual stable density is obtained when $\theta=1$ and (4.6) is a tilted density in the sense that it is proportional to $\exp \left\{-z(\theta-1)^{2} / 8\right\}$ times the stable density. See [12] XIII, §11, Problem 5 for an early derivation. $Z(t)$ is distributed as the first passage time

$$
T_{t}=\inf \left\{u>0 ; B(u)+\frac{|\theta-1|}{2} u=t\right\}
$$


where $\{B(u), u \geq 0\}$ is standard Brownian motion. The eigenvalues of $\widetilde{X}(t)$ are

$$
\begin{aligned}
\widetilde{c}_{n}(t) & =\mathbb{E}\left[\exp \left\{-\frac{1}{2} n(n+\theta-1) Z(t)\right\}\right] \\
& =\exp \left\{-t\left[\sqrt{n(n+\theta-1)+(\theta-1)^{2} / 4}-\sqrt{(\theta-1)^{2} / 4}\right]\right\} \\
& =\exp \{-t[n+(\theta-1) / 2-|\theta-1| / 2]\} \\
& =\left\{\begin{array}{l}
\exp \{-n t\} \quad \text { if } \theta \geq 1, \\
\exp \{-n t\} \times \exp \{t(1-\theta)\} \quad \text { if } \theta<1 .
\end{array}\right.
\end{aligned}
$$

The process $\{\tilde{X}(t), t \geq 0\}$ is a jump diffusion process, discontinuous at the jumps of $\{Z(t), t \geq 0\}$. Jump sizes increase as $\theta$ decreases. If $\theta<1$ then for $n \geq 1$

$$
\mathbb{E}\left[\exp \left\{-\frac{1}{2} n(n+\theta-1) Z(t)\right\}\right]=\exp \{-n t\} \times \exp \{t(1-\theta)\},
$$

so subordination does not directly produce eigenvalues $e^{-n t}$. Let $\widetilde{f}(x$, $y ; t)$ be the transition density of $\widetilde{X}(t)$, then the transition density with eigenvalues $\exp \{-n t\}, n \geq 0$ is

$$
e^{-t(1-\theta)} \tilde{f}(x, y ; t)+\left(1-e^{-t(1-\theta)}\right) f_{\alpha \beta}(y) .
$$

The subordinated process with this transition density is $X(\widehat{Z}(t))$, where $\widehat{Z}(t)$ is a similar process to $Z(t)$ but has an extra state infinity. $Z(t)$ is killed by a jump to infinity at a rate $(1-\theta)$. Another possible construction does not kill the process $\widetilde{X}$, but restarts it in a stationary state drawn from the Beta distribution. It is convenient to use the notation that a process $\left\{Z^{\circ}(t), t \geq 0\right\}$ is $\{Z(t), t \geq 0\}$ if $\theta \geq 1$, or $\{\widehat{Z}(t), t \geq 0\}$ if $0<\theta<1$, and use the single notation $\left\{X\left(Z^{\circ}(t)\right), t \geq 0\right\}$ for the subordinated process. The transition density (3.6), where $c_{n}(t)$ has the general form

$$
\exp \left\{-t \int_{0}^{\infty} \frac{\left(1-e^{-n y}\right)}{y} H(d y)\right\},
$$

can then be obtained by a composition of subordinators from the Jacobi diffusion with any $\alpha, \beta>0$.

There is a question as to which processes with transition densities (3.6) and eigenvalues $c_{n}(t)$ described by (3.7) are subordinated Jacobi diffusion processes. We briefly consider this question. Substituting

$$
R_{n}^{(\alpha, \beta)}(y)={ }_{2} F_{1}(-n, n+\theta-1 ; \beta ; 1-y)
$$


in the eigenvalue expression (3.7),

$$
\begin{aligned}
& \int_{0}^{1-} \frac{1-R_{n}^{(\alpha, \beta)}(y)}{1-y} \nu(d y) \\
& =-c \sum_{k=1}^{n} \frac{(-n)_{(k)}(n+\theta-1)_{(k)}}{\beta_{(k)}} \frac{\mu_{k-1}}{k !} \\
& =-c \sum_{k=1}^{n} \frac{\prod_{j=0}^{k-1}(-n(n+\theta-1)+j(j+\theta-1))}{\beta_{(k)}} \frac{\mu_{k-1}}{k !},
\end{aligned}
$$

where $\int_{0}^{1-}(1-y)^{k} \nu(d y)=c \mu_{k}$. The generator corresponding to a process with these eigenvalues is

$$
\widehat{\mathcal{L}}=c \sum_{k=1}^{\infty} \frac{\prod_{j=0}^{k-1}(2 \mathcal{L}+j(j+\theta-1))}{\beta_{(k)}} \frac{\mu_{k-1}}{k !},
$$

where $\mathcal{L}$ is the Jacobi diffusion process generator (1.1). The structure of the class of stochastic processes with the generator $\widehat{\mathcal{L}}$ needs to be understood better. It includes all subordinated Jacobi diffusion processes, but it seems to be a bigger class. A process with generator $\widehat{\mathcal{L}}$ is a subordinated Jacobi diffusion process if and only if the first derivative of

$$
-\sum_{k=1}^{\infty} \frac{\prod_{j=0}^{k-1}(-2 \lambda+j(j+\theta-1))}{\beta_{(k)}} \frac{\mu_{k-1}}{k !}
$$

is a completely monotone function of $\lambda$. Factorizing

$$
-2 \lambda+j(j+\theta-1)=\left(j+r_{1}(\lambda)\right)\left(j+r_{2}(\lambda)\right),
$$

where $r_{1}(\lambda), r_{2}(\lambda)$ are

$$
(\theta-1) / 2 \pm \sqrt{2 \lambda+(\theta-1)^{2} / 4},
$$

(4.8) is equal to

$$
-\int_{0}^{1-}\left[{ }_{2} F_{1}\left(r_{1}(\lambda), r_{2}(\lambda) ; \beta ; 1-y\right)-1\right](1-y)^{-1} \nu(d y) .
$$

\section{Subordinated coalescent process}

Subordinating the Jacobi diffusion process $\{X(t), t \geq 0\}$ leads to subordinating the coalescent dual process, which we investigate in this section. A subordinated process $\{\tilde{X}(t)=X(Z(t)), t \geq 0\}$ has a similar form for the transition density as (2.1), with $q_{l}^{\theta}(t)$ replaced by $\mathbb{E}\left(q_{l}^{\theta}(Z(t))\right.$, which 
are transition functions of the subordinated death process $A^{\theta}(Z(t))$. The subordinated process comes from subordinating the forest of non-mutant lineages in a coalescent tree.

If $\widetilde{A}^{\theta}(t)=A^{\theta}\left(Z^{\circ}(t)\right)$, with $Z^{\circ}(t)$ defined in the last section, we will show that the probability distribution of $\widetilde{A}^{\theta}(t), \theta>0$ is

$$
\left(\begin{array}{c}
2 k+\theta-1 \\
k
\end{array}\right)\left(\frac{z}{1+z}\right)^{k}\left(\frac{1}{1+z}\right)^{k+\theta}(1-z),
$$

for $k \in \mathbb{Z}_{+}$, where $z=e^{-t}$. The distribution (5.1) is the distribution of the number of edges in a time-subordinated forest. Note that if $0<\theta<1$ we still invoke a subordinator with a possible jump to infinity at rate $1-\theta$, so

$$
\mathbb{E}\left[q_{k}^{\theta}\left(Z^{\circ}(t)\right)\right]=e^{-(1-\theta) t} \mathbb{E}\left[q_{k}^{\theta}(Z(t))\right]+\left(1-e^{-(1-\theta) t}\right) \delta_{k 0},
$$

because $q_{k}^{\theta}(\infty)=\delta_{k 0}$. Although $\theta$ is greater than zero in (5.1), it is interesting to consider the subordinated Kingman coalescent with no mutation. Then $A^{0}(t) \geq 1$, and

$$
\mathbb{E}\left[q_{k}^{0}\left(Z^{\circ}(t)\right)\right]=e^{-t} \mathbb{E}\left[q_{k}^{0}(Z(t))\right]+\left(1-e^{-t}\right) \delta_{k 1},
$$

because a jump to infinity is made at rate 1 , and $q_{k}^{\circ}(\infty)=\delta_{k 1}$. The distribution of $\widetilde{A}^{0}(t)$ is then, for $k \geq 1$,

$$
\left(\begin{array}{c}
2 k-1 \\
k
\end{array}\right)\left(\frac{z}{1+z}\right)^{k}\left(\frac{1}{1+z}\right)^{k}(1-z)+\delta_{k 1}(1-z) .
$$

The proof of (5.1) $(\theta>0)$ and (5.2) (with $\theta=0)$ follows directly from the expansion (2.2).

$$
\begin{aligned}
\mathbb{E}\left[q_{k}^{\theta}\left(Z^{\circ}(t)\right)\right]= & \sum_{j=k}^{\infty} z^{j}(-1)^{j-k} \frac{(2 j+\theta-1)(k+\theta)_{(j-1)}}{k !(j-k) !} \\
= & \frac{\Gamma(2 k+\theta)}{k ! \Gamma(k+\theta)} z^{k} \\
& \times\left\{1+\sum_{j=1}^{\infty}(-1)^{j}(2 j+2 k+\theta-1) \frac{(2 k+\theta)_{(j-1)}}{j !} z^{j}\right\} \\
= & \frac{\Gamma(2 k+\theta)}{k ! \Gamma(k+\theta)} z^{k}(1-z)(1+z)^{-(2 k+\theta)} \\
= & \left(\begin{array}{c}
2 k+\theta-1 \\
k
\end{array}\right)\left(\frac{z}{1+z}\right)^{k}\left(\frac{1}{1+z}\right)^{k+\theta}(1-z) .
\end{aligned}
$$

Effectively, in the expansion (2.2) of $q_{k}^{\theta}(t)$, terms $\rho_{j}(t)=\exp \left\{-\frac{1}{2} j(j+\right.$ 
$\theta-1) t\}$ are replaced by $z^{j}=\exp \{-j t\}$. The third line of (5.3) follows from the identity, with $|z|<1$ and $\alpha=2 k+\theta$, that

$$
(1-z)(1+z)^{-\alpha}=1+\sum_{j=1}^{\infty}(-1)^{j}(2 j+\alpha-1) \frac{\alpha_{(j-1)}}{j !} z^{j},
$$

proved by equating coefficients of $z^{j}$ on both sides. Of course, for any $|z|<1$, since (5.1) is a probability distribution,

$$
\sum_{k=0}^{\infty}\left(\begin{array}{c}
2 k+\theta-1 \\
k
\end{array}\right)\left(\frac{z}{1+z}\right)^{k}\left(\frac{1}{1+z}\right)^{k+\theta}(1-z)=1 .
$$

The probability generating function of (5.1) is

$$
G_{\widetilde{A}^{\theta}(t)}(s)=\left(\frac{1-4 p q s}{1-4 p q}\right)^{-\frac{1}{2}}\left(\frac{1-\sqrt{1-4 p q s}}{2 p s}\right)^{\theta-1}, \theta>0,
$$

where $p=e^{-t} /\left(1+e^{-t}\right)$ and $q=1 /\left(1+e^{-t}\right)$. The calculation needed to show (5.5) comes from the identity

$$
\sum_{k=0}^{\infty}\left(\begin{array}{c}
2 k+\theta-1 \\
k
\end{array}\right) w^{k}=2^{\theta-1} \frac{(1+\sqrt{1-4 w})^{-(\theta-1)}}{\sqrt{1-4 w}}
$$

which is found by substituting

$$
w=\frac{z}{(1+z)^{2}} \text { or } z=\frac{1-\sqrt{1-4 w}}{1+\sqrt{1-4 w}}
$$

in (5.4), then setting

$$
w=\frac{s z}{(1+z)^{2}}
$$

in (5.6). The calculations used in obtaining the distribution and probability generating function are the same as those used in obtaining the formula (2.3) in Griffiths [17]. There is a connection with a simple random walk on $\mathbb{Z}$ with transitions $j \rightarrow j+1$ with probability $p$ and $j \rightarrow j-1$ with probability $q=1-p$, when $q \geq p$. Let the number of steps to hit $-\theta$, starting from 0 , be $\xi$. Then $\xi$ has a probability generating function of

$$
H(s)=\left(\frac{1-\sqrt{1-4 p q s^{2}}}{2 p s}\right)^{\theta},
$$

and $\frac{1}{2}(\xi+\theta)$ has a probability generating function

$$
K(s)=\left(\frac{1-\sqrt{1-4 p q s}}{2 p}\right)^{\theta} .
$$


$\widetilde{A}^{\theta}(t)+\theta$ has the same distribution as the size-biased distribution of $\frac{1}{2}(\xi+\theta)$, with probability generating function

$$
G_{\widetilde{A}^{\theta}(t)}(s)=\frac{s K^{\prime}(s)}{K^{\prime}(1)},
$$

identical to (5.5). In the random walk interpretation $\theta$ is assumed to be an integer; however $H(s)$ is infinitely divisible, so we use the same description for all $\theta>0$. Another interpretation is that $K(s)$ is the probability generating function of the total number of progeny in a Galton-Watson branching process with geometric offspring distribution $q p^{k}, k \in \mathbb{Z}_{+}$, and extinction probability 1 , beginning with $\theta$ individuals. See 11] Sections X.13 and XII.5 for details of the random walk and branching process descriptions. An analogous calculation to (5.3) which is included in Theorem 2.1 of [17] is that

$$
\begin{aligned}
\mathbb{P}\left(\widetilde{A}^{\theta}(s+t)=j \mid \widetilde{A}^{\theta}(s)=i\right) \\
=\left(\begin{array}{c}
i \\
j
\end{array}\right) \frac{\Gamma(i+\theta) \Gamma(2 j+\theta)}{\Gamma(j+\theta) \Gamma(i+j+\theta)} z^{j}(1-z) \\
\quad \times{ }_{2} F_{1}(-i+j+1,2 j+\theta ; i+j+\theta ; z),
\end{aligned}
$$

where $z=e^{-t}$. The jump rate from $i \rightarrow j$ found from (5.7) is

$$
\begin{aligned}
\left(\begin{array}{l}
i \\
j
\end{array}\right) & \frac{\Gamma(i+\theta) \Gamma(2 j+\theta)}{\Gamma(j+\theta) \Gamma(i+j+\theta)}{ }_{2} F_{1}(-i+j+1,2 j+\theta ; i+j+\theta ; 1), \\
= & \left(\begin{array}{l}
i \\
j
\end{array}\right) B(j+\theta, i-j)^{-1} \int_{0}^{1} x^{2 j+\theta-1}(1-x)^{2(i-j)-2} d x \\
= & \left\{\begin{array}{l}
(i) \frac{\Gamma(2 i-2 j-1) \Gamma(2 j+\theta) \Gamma(i+\theta)}{\Gamma(-j) \Gamma(j+\theta) \Gamma(2 i+\theta-1)} \quad \text { if } j=i-1, i-2, \ldots, \\
\frac{\Gamma(2 j+\theta)}{\Gamma(j+\theta) j !}\left(\frac{1}{2}\right)^{2 j+\theta} \text { if } i=\infty .
\end{array}\right.
\end{aligned}
$$

Bertoin [4], [5] studies the genealogical structure of trees in an infinitelymany-alleles branching process model. In a limit from a large initial population size with rare mutations the genealogy is described by a continuous-state branching process in discrete time with an Inverse Gaussian reproduction law. We expect that there is a fascinating connection with the process $\left\{\widetilde{A}^{\theta}(t), t \geq 0\right\}$. A potential class of transition functions of Markov processes $\left\{\widehat{q}_{k}^{\theta}(t), t \geq 0\right\}$ which are more general than subordinated processes and related to Bochner's characterization comes from replacing by $\rho_{n}^{\theta}(t)$ by $c_{n}(t)$ described by (3.7); however it is not clear that all such potential transition functions are positive, apart from those derived by subordination. 


\section{References}

[1] Andrews, G. E., Askey, R., and Roy, R. 1999. Special Functions. Encyclopedia Math. Appl., vol. 71. Cambridge: Cambridge Univ. Press.

[2] Bailey, W. N. 1938. The generating function of Jacobi polynomials. J. Lond. Math. Soc., 13, 8-11.

[3] Barbour, A. D., Ethier, S. N., and Griffiths, R. C. 2000. A transition function expansion for a diffusion model with selection. Ann. Appl. Probab., 10, 123-162.

[4] Bertoin, J. 2009. The structure of the allelic partition of the total population for Galton-Watson processes with neutral mutations, Ann. Probab., 37, 1052-1523.

[5] Bertoin, J. 2010. A limit theorem for trees of alleles in branching processes with rare mutations, Stochastic Process. Appl., to appear.

[6] Bochner, S. 1954. Positive zonal functions on spheres. Proc. Natl. Acad. Sci. USA, 40, 1141-1147.

[7] Donnelly, P. J., and Kurtz, T. G. 1996. A countable representation of the Fleming-Viot measure-valued diffusion. Ann. Appl. Probab., 24, 698-742.

[8] Donnelly, P. J., and Kurtz, T. G. 1999. Particle representations for measure-valued population models. Ann. Probab., 24, 166-205.

[9] Etheridge, A. M., and Griffiths, R. C. 2009. A coalescent dual process in a Moran model with genic selection, Theor. Popul. Biol., 75, 320-330.

[10] Ethier, S. N., and Griffiths, R. C. 1993. The transition function of a Fleming-Viot process. Ann. Probab., 21, 1571-1590.

[11] Feller, W., 1968. An Introduction to Probability Theory and its Applications, vol. I, 3rd edn. New York: John Wiley \& Sons.

[12] Feller, W., 1971. An Introduction to Probability Theory and its Applications, vol. II, 2nd edn. New York: John Wiley \& Sons.

[13] Gasper, G. 1972. Banach algebras for Jacobi series and positivity of a kernel. Ann. of Math. (2), 95, 261-280.

[14] Griffiths, R. C. 1970. Positive definite sequences and canonical correlation coefficients. Austral. J. Statist., 12, 162-165.

[15] Griffiths, R. C. 1979. A transition density expansion for a multi-allele diffusion model. Adv. in Appl. Probab., 11, 310-325.

[16] Griffiths, R. C. 1980. Lines of descent in the diffusion approximation of neutral Wright-Fisher models. Theor. Popul. Biol., 17, 37-50.

[17] Griffiths, R. C. 2006. Coalescent lineage distributions. Adv. in Appl. Probab., 38, 405-429.

[18] Griffiths, R. C. [Griffiths, B.] 2009. Stochastic processes with orthogonal polynomial eigenfunctions. J. Comput. Appl. Math., 23, 739-744.

[19] Kingman, J. F. C. 1982. The coalescent. Stochastic Process. Appl., 13, $235-248$.

[20] Kingman, J. F. C. 1993. Poisson Processes. Oxford: Oxford Univ. Press.

[21] Tavaré, S. 1984. Line-of-descent and genealogical processes, and their application in population genetics models. Theor. Popul. Biol., 26, 119164. 